\title{
The Association between Health-related Quality of Life and Depression on Activity Restriction in Osteoarthritis: A Cross-sectional Study
}

\author{
Do-Youn Lee', Seong-Gil Kim² \\ 'Department of Physical Therapy, Daegu University, Daegu, Republic of Korea; ${ }^{2}$ Department of Physical Therapy, Sun Moon University, Asan, \\ Republic of Korea
}

Purpose: The purpose of this study is to provide basic evidence on the need to approach osteoarthritis patients through a psychological factors-considering rehabilitation program by understanding how activity restrictions in osteoarthritis affect health-related quality of life and depression.

Methods: This study assessed 3,761 osteoarthritis patients from the Korea National Health and Nutrition Examination Survey. The subjects were divided into two categories: with and without activity restriction.

Results: The prevalence of osteoarthritis in women was higher than that of men (men: 19.7\%; women: 80.3\%), and high BMI increased the prevalence of osteoarthritis. The EQ-5D index of subjects with activity restriction was $0.84 \pm 0.18$ (points), while in those without activity restriction was $0.93 \pm 0.12$, and the diagnosis of depression was $15.8 \%, 8.2 \%$. There was a statistically significant difference in the odds ratio for each item in the EQ-5D. Moreover, the odds ratio for depression with activity restriction was 2.098 compared with no activity restriction.

Conclusion: Activity restriction of osteoarthritis patients significantly decreases the health-related quality of life and increase the probability of depression. Therefore, early diagnosis of depression symptoms to prevent deterioration of symptoms in patients with osteoarthritis and to increase compliance with rehabilitation treatment, and to provide arbitration, including treatment that can alleviate depression.

Keywords: Osteoarthritis, Health-related quality of life, Depression

\section{INTRODUCTION}

Arthritis is the main cause of limiting physical activity in patients. ${ }^{1}$ The most osteoarthritis among arthritis is a typical chronic degenerative disease in Korea, with an annual medical cost of 6.44 million won per person, accounting for the largest portion of the chronic disease's medical expenses. ${ }^{2,3}$ In addition, the prevalence of osteoarthritis is on the rise with the aging population, which is a condition that brings pain and physical disability and is expected to increase medical spending. ${ }^{4}$ In particularly, knee osteoarthritis has been found to be a major factor causing long-term walking exercise and functional disorders in the body of the elderly. ${ }^{5,6}$

Osteoarthritis is not a life-threatening condition and is often not sub- ject to initial management and appropriate medical treatment because it is considered a process of normal aging. ${ }^{6,7}$ This not only results in symptoms such as joint friction, joint pain, and stiffness along with pathological changes such as damage to joint cartilage, but also experiences limitations of functional activity. ${ }^{8,9}$

Quality of life is an assessment of the degree and value of life centered on an individual's socio-cultural and environmental backgrounds and is a long-term outcome assessment index used in patients with various chronic diseases, such as arthritis and diabetes. ${ }^{10-15}$ Most elderly people with osteoarthritis experience physical and mental health problems and low health-related quality of life. ${ }^{16}$ According to a prior study, older people with osteoarthritis perceive their health conditions worse than those with- 
out osteoarthritis. ${ }^{17}$ Moreover, patients with osteoarthritis experience more depression than healthy elderly people or those without arthritis as they experience walking disorders, restrictions on daily activities and falls due to symptoms such as pain and stiffness caused by disease. ${ }^{18-20}$

Depression has a negative effect on the recovery of dysfunction because it leads to a loss of roles, and in severe cases, it reduces the motivation of rehabilitation, making treatment difficult, directly affecting physical functional conditions and poor quality of life. ${ }^{21}$ In patients with osteoarthritis, depression can also have a negative effect on the intervention of pain management or post-operative outcomes, ${ }^{22}$ aggravating pain, and increasing the risk of suicide. ${ }^{23}$

According to the prior study, there were many studies on the quality of life and the degree of depression in patients with osteoarthritis, but studies on the direct influence of the restriction of activity in patients with osteoarthritis on quality of life and depression were not active. ${ }^{3,16,17}$ Therefore, this study was conducted on patients with osteoarthritis to find specific influences on the health-related quality of life and the relevance of depression with or without restrictions on activity. By identifying these associations, this study also seeks to provide health care professionals involved in treatment with basic evidence on the need to approach osteoarthritis patients by taking into account psychological factors in their treatment and rehabilitation programs, and to assist in the clinical direction of intervention that can alleviate depression and improve the quality of life.

\section{METHODS}

\section{Data source and sampling}

This study used data from the 6th and 7th Korea National Health and Nutrition Examination Survey (KNHANES, 2013-2018) conducted by the Korea Centers for Disease Control and Prevention. Out of a total of 47,217 people, 26,462 people under 50 years of age, 2,956 non-response to diagnosis of osteoarthritis, and 429 non-response to diagnosis of healthrelated quality of life and depression were excluded. Finally, 17,370, of which 3,761 were diagnosed with osteoarthritis, and 13,662 were not diagnosed.

\section{Measurements of variables}

In this study, the health survey items of KNHANES were analyzed using data from gender, age, personal income level, marital status, body mass index (BMI), smoking and drinking status, subjective health condition, activity restriction, health-related quality of life survey, and diagnosis of de- pression.

Age was calculated as the average age value based on the data, and individual income levels were divided into "Quartile 1: Lowest" to "Quartile 4: Highest" using income classification based on the quartile. In the case of marriage, it was reclassified as "yes" if the patient lived with a spouse and "no" if the patient did not.

BMI was reclassified as "low weight: $\mathrm{BMI}<18.5$ ", "normal: $\geqq 18.5,<25$ ”, and "overweight: $\geqq 25$ " using variables calculated as [weight $(\mathrm{kg}) /$ height $\left(\mathrm{m}^{2}\right)$ ]. Smoking conditions were classified as "current", "former" and "never". Drinking conditions were classified as "no" in the latest year's frequency of drinking, when people answered "no more than once a month or never drank" and "yes" if the answer was "one or four times a month, two to four times a month, or two to three times a week or more". Subjective health conditions were divided into "good", "normal" and "bad", and the diagnosis of depression used the responses "yes" and "no".

The health-related quality of life for osteoarthritis patients was analyzed using EQ-5D (EuroQol-5 Dimension) (Figure 1). EQ-5D has been developed as a tool to measure the health-related quality of life and is divided into five areas: mobility, self-care, usual activities, pain/discomfort, anxiety/depression. The EQ-5D indicators of health-related quality of life that

\section{EQ-5D Health Questionnaire}

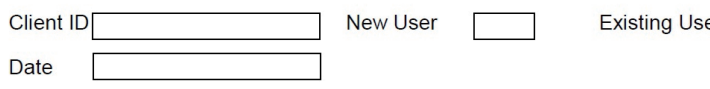

By placing a tick in one box in each group below, please indicate which statements best describe your own health state today.

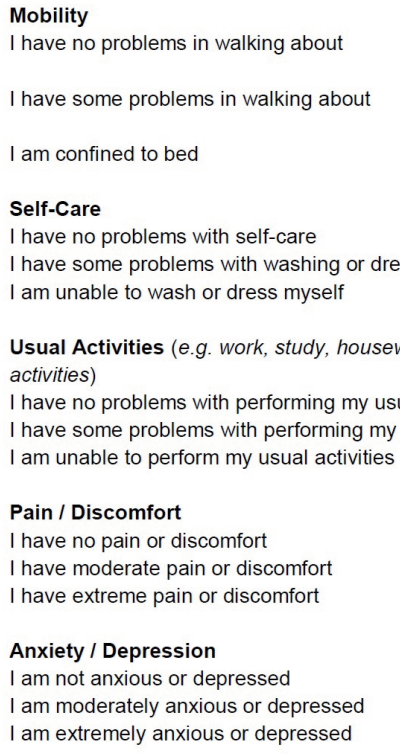

Figure 1. EQ-5D Health Questionnaire. 
are used for chronic patients consist of three levels: "no problems", "moderate problems", and "severe problems". These are weighted on each of the measures of five problems. This value ranges from 1 for complete health to -1 for less than death. In this study, three levels of answers were divided into two groups (No problem/problem present). The problem group included those who responded to 'moderate problem' and 'severe problem' among the three levels of severity.

\section{Statistical analysis}

The data of KNHANES were weighted in consideration of the extraction rate and response rate so that the subjects participating in the survey could represent the people of Korea, and in this study, the "itvex" weight was reflected in the data analysis. The characteristics of the subjects were analyzed using descriptive statistics. An independent t-test was used to compare differences in the mean values of baseline parameters among the groups. Moreover, subjects' depression and health-related quality of life were analyzed using chi-square test according to their activity limitation. Using logistic regression analysis, gender, age, personal income level, marital status, BMI, smoking and drinking status, and subjective health status were adjusted and analyzed for the link between the activity limit of the subjects and the quality of life related to depression and health. The data analysis used the SPSS 22.0 program and the statistical significance level was defined as $\mathrm{p}<0.05$.

\section{RESULTS}

The prevalence of osteoarthritis in women was higher than that of men (men: 19.7\%; women: 80.3\%), and high BMI increased the prevalence of osteoarthritis. In addition, patients with osteoarthritis responded that their subjective health conditions were poor and that there were restrictions on their activities. The diagnosis of depression was more than double the rate of osteoarthritis patients compared to normal (Table 1).

The quality of health-related life of patients with osteoarthritis was statistically significantly lower than 0.93 of those who responded that there was no activity restriction, with 0.84 of those who responded that there was an activity restriction based on a maximum value of 1 . Each element of quality of life was divided into mobility, self-care, usual activities, pain/ discomfort, anxiety/depression, and showed statistically significant differ-

Table 1. Characteristics of the study population aged $\geqq 50$ years of KNHANES, 2013 to 2018

\begin{tabular}{|c|c|c|c|c|}
\hline \multirow{2}{*}{ Characteristics } & \multirow{2}{*}{ Categories } & \multicolumn{2}{|c|}{$\mathrm{OA}$} & \multirow{2}{*}{$\mathrm{p}$} \\
\hline & & Yes $(n=3,761)$ & No $(n=13,662)$ & \\
\hline Age (yr) & & $66.85 \pm 0.17$ & $61.39 \pm 0.09$ & $<0.0001$ \\
\hline \multirow[t]{2}{*}{$\operatorname{Sex}(n, \%)$} & Male & $694(19.7)$ & $6,756(53.0)$ & $<0.0001$ \\
\hline & Female & $3,067(80.3)$ & $6,906(47.0)$ & \\
\hline \multirow[t]{4}{*}{ Income (individual) } & Quartile 1 (lowest) & $1,051(29.0)$ & $3,179(23.3)$ & \\
\hline & Quartile 2 & $980(25.3)$ & $3,399(24.8)$ & \\
\hline & Quartile 3 & $887(23.0)$ & $3,459(25.1)$ & \\
\hline & Quartile 4 (highest) & $843(22.7)$ & $3,625(26.8)$ & \\
\hline \multirow[t]{2}{*}{ Spouse (n, \%) } & Yes & $2,378(64.0)$ & $10,797(80.4)$ & $<0.0001$ \\
\hline & No & $1,383(36.0)$ & $2,865(19.5)$ & \\
\hline \multirow[t]{3}{*}{$\mathrm{BMI}\left(\mathrm{kg} / \mathrm{m}^{2}, \%\right)$} & Underweight $(<18.5)$ & $48(1.2)$ & $377(2.6)$ & $<0.0001$ \\
\hline & Normal $(\geqq 18.5,<25)$ & $1,950(52.1)$ & $8,654(62.8)$ & \\
\hline & Overweight ( $\geqq 25$ ) & $1,763(46.7)$ & $4,631(34.6)$ & \\
\hline \multirow[t]{3}{*}{ Smoking status (n, \%) } & Current & $244(7.5)$ & $2,222(18.5)$ & $<0.0001$ \\
\hline & Former & $536(15.0)$ & $3,807(28.4)$ & \\
\hline & Never & 2,981 (77.5) & $7,633(53.1)$ & \\
\hline \multirow[t]{2}{*}{ Alcohol drinking status (n, \%) } & Yes & $1,845(51.1)$ & $8,781(67.2)$ & $<0.0001$ \\
\hline & No & $1,916(48.9)$ & $4,881(32.8)$ & \\
\hline \multirow[t]{3}{*}{ Subject health condition (n, \%) } & Good & $471(12.6)$ & $3,709(28.0)$ & $<0.0001$ \\
\hline & Ordinary & $1,722(46.7)$ & $7,048(52.0)$ & \\
\hline & Poor & $1,568(40.7)$ & $2,905(20.0)$ & \\
\hline Depression (n, \%) & Diagnosed by a doctor & $383(9.9)$ & $640(4.4)$ & $<0.0001$ \\
\hline Activity limitation (n, \%) & Yes & $890(22.8)$ & $1400(9.4)$ & $<0.0001$ \\
\hline
\end{tabular}

$\mathrm{M} \pm \mathrm{SD}$ : mean \pm standard deviation, BMl: body mass index, $\mathrm{p}$ value for t-test or Rao-scott chi-square test. 
Table 2. Proportion of depression and health-related quality of life according to the activity limitation of osteoarthritis patients

\begin{tabular}{|c|c|c|}
\hline Characteristics / Categories & $\begin{array}{c}\text { Activity limitation } \\
\text { Yes }(n=890)\end{array}$ & $\begin{array}{l}{[\mathrm{M} \pm \mathrm{SD} \text { or } \%]} \\
\text { No }(n=2,871)\end{array}$ \\
\hline \multicolumn{3}{|l|}{ EQ-5D } \\
\hline Index (points) ${ }^{*}$ & $0.84 \pm 0.18$ & $0.93 \pm 0.12$ \\
\hline Problem of mobility* & $652(71.7)$ & $1,180(39.1)$ \\
\hline Problem of self-care* & $260(27.9)$ & $246(7.8)$ \\
\hline Problem of usual activities* & $510(55.4)$ & $560(17.9)$ \\
\hline Problem of pain/discomfort* & $702(78.8)$ & $1,288(44.4)$ \\
\hline Problem of anxiety/depression* & $365(38.9)$ & $499(16.8)$ \\
\hline \multicolumn{3}{|l|}{ Subject health status* } \\
\hline Good & $40(4.8)$ & $431(14.9)$ \\
\hline Ordinary & $246(28.1)$ & $1,476(52.2)$ \\
\hline Poor & $604(67.1)$ & 964 (32.9) \\
\hline \multicolumn{3}{|l|}{ Depression (diagnosed by a doctor)* } \\
\hline Yes & $145(15.8)$ & $238(8.2)$ \\
\hline
\end{tabular}

by chi-square test, $\mathrm{M} \pm \mathrm{SD}$ : mean \pm standard deviation. ${ }^{*} p<0.0001$.

ences in each item depending on whether the activity was restricted. In addition, subjective health conditions showed $67.1 \%$ of those with activity restrictions answered they were "poor," while 52.0\% answered they were "ordinary" to those without activity restrictions. The diagnosis of depression showed a statistically significant difference of $15.8 \%$ in groups with activity restrictions and $8.2 \%$ in groups without activity restrictions (Table 2).

In odds ratio of health-related quality of life after adjusted by gender, age, marriage, BMI, smoking and drinking, and subjective health conditions, there were problems of 3.935 in mobility, 4.129 in self-care, 5.470 in daily activities, 4.584 in pain/discomfort, and 3.069 in anxiety/depression (Table 3).

The diagnosis of depression in those who responded with activity restrictions was 2.098 times higher than those who did not have activity restrictions (Table 4).

\section{DISCUSSION}

Diseases with high incidence, such as osteoarthritis, that require continuous medical services and high medical spending need to identify the health-related quality of life of the recipient. This study, using data from the 6th and 7th KNHANES, looked at the effects of activity restrictions on the health-related quality of life and depression of osteoarthritis patients. Comparing the relationship between quality of life and depression by stratifying the subjects of this study according to their activity restric-
Table 3. Association between each item of EQ-5D and activity limitation in osteoarthritis patients

\begin{tabular}{|c|c|c|}
\hline \multirow{2}{*}{ Individual components } & \multicolumn{2}{|c|}{ Activity limitation, OR $(95 \% \mathrm{Cl})$} \\
\hline & Yes & No \\
\hline \multicolumn{3}{|l|}{ EQ-5D } \\
\hline Problem of mobility* & $3.935(3.207-4.828)$ & 1 (ref) \\
\hline Problem of self-care* & $4.129(3.259-5.232)$ & 1 (ref) \\
\hline Problem of usual activities* & $5.470(4.487-6.668)$ & $1(\mathrm{ref})$ \\
\hline Problem of pain/discomfort* & $4.584(3.708-5.667)$ & 1 (ref) \\
\hline Problem of anxiety/depression* & $3.069(2.524-3.732)$ & 1 (ref) \\
\hline
\end{tabular}

$\mathrm{p}$ values were obtained by logistic regression analysis adjusted for sex, age, spouse, BMI, smoking status, alcohol drinking status, subject health status. $* p<0.0001$.

Table 4. Association between depression diagnosis and activity limitation in osteoarthritis patients

\begin{tabular}{lcc}
\hline \multirow{2}{*}{ Depression } & \multicolumn{2}{c}{ Activity limitation, OR $(95 \% \mathrm{Cl})$} \\
\cline { 2 - 3 } & Yes & No \\
\hline diagnosed by a doctor* & $2.098(1.613-2.728)$ & 1 (ref) \\
\hline
\end{tabular}

$\mathrm{p}$ values were obtained by logistic regression analysis adjusted for sex, age, spouse, BMI, smoking status, alcohol drinking status, subject health status. $\star p<0.0001$.

tions, there was a significant difference.

Comparing health-related life quality relationships under activity restrictions, EQ-5D index showed a significant difference of 0.84 points for those who said they had activity restrictions and 0.93 points for those who said they did not. Individual items also showed statistically significant differences in mobility, self-care, daily activities, pain/discomfort, anxiety/ depression. The impact of activity restrictions on the quality of life also showed that those who responded with "problem present" were 3.94 times, 4.13 times, 5.47 times, 4.58 times and 3.07 times higher for each category compared to "no problem." In addition, the number of cases in which subject's health is perceived to be poor under subjective health conditions more than doubled to $32.9 \%$ in groups without activity restrictions and $67.1 \%$ in groups with activity restrictions. These results indicate that osteoarthritis patients with activity restrictions have significantly lower quality of health-related life compared to those without activity restrictions, and serious problems with mobility, self-care, daily activities, pain/discomfort, anxiety/depression, and subjective health conditions.

This is in line with the results of a prior study that, in the case of elderly osteoarthritis patients, the quality of life decreases significantly due to persistent joint pain, fatigue, and physical activity restrictions, ${ }^{24}$ and when they feel there are many restrictions on behavior or restrictions on social roles, the quality of life can be further decreased. ${ }^{25}$ Also, knee rigidity, the main symptom of osteoarthritis, is consistent with the results of a prior 
study that people tend to perceive their health conditions as worse than patients without disabilities because it limits the patients' social and daily activities by causing walking disorders and physical disabilities. ${ }^{8,17}$

The diagnosis of depression in those subject to osteoarthritis with activity restrictions was 15.8 percent, significantly higher than 8.2 percent in those without activity restrictions. The comparison of influence between the two groups showed 2.098 times higher than those without activity restrictions. These findings suggest that the activity restrictions and depression of osteoarthritis patients are positively correlated.

This association of activity restrictions and depression diagnoses in this study shows that functional activity restrictions and subjective health conditions are consistent with the results of studies that have been shown as factors of influence closely related to depression in older Japanese people with arthritis. ${ }^{26}$ In addition, another studies reported that arthritis has a direct impact on depression show significant differences even after adjusted by other chronic and functional conditions (smoking, drinking, cognitive levels, subjective health conditions, antidepressants, and the number of chronic diseases). ${ }^{27}$

Elderly with osteoarthritis often experience isolation due to chronic pain and difficulty in social life caused by walking disorder compared to healthy old people, ${ }^{28}$ and their stress levels can increase due to the repetition and deterioration of osteoarthritis symptoms and the economic and psychological burden of ongoing management for this purpose. ${ }^{29}$ Osteoarthritis patients with negative emotions, such as depression due to restrictions on daily activities, are known to not only have higher appeals for pain compared to patients who do not, ${ }^{8}$ but also to have less participation and adaptability to rehabilitation, such as pain arbitration. ${ }^{22}$ Furthermore, the deterioration in health-related quality of life due to psychological problems of the elderly with osteoarthritis should be more thoroughly managed because there is a risk of leading to suicide accidents of the elderly. ${ }^{23}$

The advantages of this study are significant clinical and research implications because it is a large sample of patients that can represent the Korean population, and because it has been systematically performed by experts, various statistical adjustments are possible to enhance the validity and sensitivity of the study. The additional strength of this study is that it is possible to more comprehensively analyze the relationship between activity restrictions and health-related quality of life and depression in osteoarthritis patients, while calibrating various potential confounding (sex, age, marital status, BMI, smoking and drinking status, subjective health status). However, since this is a study using sample data, there are limita- tions that are difficult to explain the context of time and potential sampling error as survey studies. Therefore, it is believed that more comprehensive research is needed in the future by conducting a longitudinal trace survey to compensate for these shortcomings.

This study looked at the effects of activity restriction in osteoarthritis on health-related quality of life and depression. The results of the study showed that patients with osteoarthritis with restricted activity had lower quality of life and higher rates of diagnosis of depression. These results require early diagnosis of depression symptoms to prevent deterioration of symptoms in patients with osteoarthritis and to increase compliance with rehabilitation treatment, and to provide arbitration, including treatment that can alleviate depression. Furthermore, osteoarthritis causes longterm pain for individuals along with significant socioeconomic costs, patient care systems will need to be specifically established through ongoing follow-up, along with training on arthritis management and joint strengthening exercises to improve health-related quality of life.

\section{REFERENCES}

1. Choi MS, Lee DK. The effect of knee joint traction therapy on pain, physical function, and depression in patients with degenerative arthritis. J Kor Phys Ther. 2019;31(5):317-21.

2. Lee EK. Impact of aging on elderly health care expenditure in korea. Korea Institute of Public Finance. 2011.

3. Park HJ, Lee SK. The association between osteoarthritis and health-related quality of life in women aged 50 years and over: Using the korea national health and nutrition examination survey (knhanes) data. Korean J Health Edu Promot. 2012;29(2):23-32.

4. Yelin E, Murphy L, Cisternas MG et al. Medical care expenditures and earnings losses among persons with arthritis and other rheumatic conditions in 2003, and comparisons with 1997. Arthritis Rheum. 2007;56 (5):1397-407.

5. Kim HR, Kim EJ. Prevalence of osteoarthritis and its affecting factors among a korean population aged 50 and over. J Korean Pubilc Health Nurs. 2013;27(1):27-39.

6. Park SJ, Lee JH. Effect of joint mobilization and kinesio taping on pain, range of motion, and knee function in patients with knee osteoarthritis. J Kor Phys Ther. 2016;28(5):279-85.

7. Kim M, Bae SH. Factors influencing health-related quality of life in older adults with osteoarthritis: Based on the 2010-2011 korea national health and nutrition examination survey. J Muscle Jt Health. 2014;21(3):195205.

8. Hunter DJ, Riordan EA. The impact of arthritis on pain and quality of life: An a ustralian survey. Int J Rheum Dis. 2014;17(2):149-55.

9. Sadosky AB, Bushmakin AG, Cappelleri JC et al. Relationship between patient-reported disease severity in osteoarthritis and self-reported pain, function and work productivity. Arthritis Res Ther. 2010;12(4):R162.

10. Jun HJ, Kim KJ, Chun IA et al. The relationship between stroke patients' 
socio-economic conditions and their quality of life: The 2010 korean community health survey. J Phys Ther Sci. 2015;27(3):781-4.

11. Kim HS. The effect of protective socks with functional insoles on plantar foot pressure in diabetes patients. J Kor Phys Ther. 2018;30(6):224-8.

12. Lee SY, Choi SH, Ha KJ et al. An investigation study on the role and performance ability of physical therapists in the community care system for elderly. J Kor Phys Ther. 2020;32(4):266-71.

13. Kim EK. Comparison of vital capacity and balance between elderly women and young women with forward head postures according to the use of figure- 8 brace. J Kor Phys Ther. 2019;31(4):248-53.

14. Kim EK, Lee DK. Effect of scapular brace on the pulmonary function and foot pressure of elderly women with forward head posture. J Kor Phys Ther. 2018;30(4):141-5.

15. Kim GE, Choi YE, Yang SS et al. Relationship between functional fitness and break response in elderly drivers: Comparison with young driver. J Kor Phys Ther. 2018;30(5):187-92.

16. Alrushud AS, El-Sobkey SB, Hafez AR et al. Impact of knee osteoarthritis on the quality of life among saudi elders: A comparative study. Saudi J Sports Med. 2013;13(1):10.

17. Yang SJ, An JS. Health status, health behavior and quality of life in the elderly with osteoarthritis. Nursing Science. 2011;23(2):23-33.

18. Vennu V, Bindawas SM. Relationship between falls, knee osteoarthritis, and health-related quality of life: Data from the osteoarthritis initiative study. Clin Interv Aging. 2014;9:793.

19. Yeo SS. Changes of gait variability by the attention demanding task in elderly adults. J Kor Phys Ther. 2017;29(6):303-6.

20. Seo SH, Bae HB, Cho YJ et al. Immediate effect of eye movement on static balance according to age in elderly individuals. J Kor Phys Ther. 2016;28(5):274-8.

21. Mesci E, Icagasioglu A, Mesci N et al. Relation of physical activity level with quality of life, sleep and depression in patients with knee osteoarthritis. North Clin Istanb. 2015;2(3):215.

22. Broderick JE, Junghaenel DU, Schneider S et al. Treatment expectation for pain coping skills training: Relationship to osteoarthritis patients' baseline psychosocial characteristics. Clin J Pain. 2011;27(4):315.

23. Lee $\mathrm{SH}$, Oh KO, Kang MH. A study on factors affecting the suicidal ideation in older adults with osteoarthritis. J Korean Acad Psychiatr Ment Health Nurs. 2011;20(1):103-10.

24. Rivard V, Cappeliez P. Perceived control and coping in women faced with activity restriction due to osteoarthritis: Relations to anxious and depressive symptoms. Can J Aging. 2007;26(3):241-53.

25. Son J. Factors influencing quality of life of patients with osteoarthritis in rural area. J Korean Geriatr Soc. 2014;34(1):23-35.

26. Nakajima A, Kamitsuji S, Saito A et al. Disability and patient's appraisal of general health contribute to depressed mood in rheumatoid arthritis in a large clinical study in japan. Mod Rheumatol. 2006;16(3):151-7.

27. Niti M, Ng TP, Kua EH et al. Depression and chronic medical illnesses in asian older adults: The role of subjective health and functional status. Int J Geriatr Psychiatry. 2007;22(11):1087-94.

28. Jakobsson U, Hallberg IR. Quality of life among older adults with osteoarthritis: An explorative study. J Gerontol Nurs. 2006;32(8):51-60.

29. Choi IS, Park K. The effects of pain, sleep, and depression on quality of life in elderly people with chronic pain. J Digit Converg. 2017;15(8):28999. 